\title{
Negative symptoms and therapy strategies in schizophrenia
}

\author{
Andrea Schmitt $^{1} \cdot$ Peter Falkai $^{1}$
}

Published online: 29 August 2015

(C) Springer-Verlag Berlin Heidelberg 2015

In schizophrenia, negative symptoms are associated with poor functional outcome and psychosocial disability. Current treatment with antipsychotics has only limited effects on this symptom domain, and its neurobiological background is only poorly understood. In a comprehensive review, Galderisi et al. [1] focused on the underlying neurobiological basis, including structural and functional neuroimaging data showing alterations in prefronto-temporoparietal brain circuits, and discuss complex alterations in neurotransmitter systems and genetic variations. However, negative symptoms are a heterogeneous symptom cluster and show also association with cognitive deficits. GarciaPortilla et al. [2] reviewed the available instruments for assessing the negative syndrome, considering strength and weaknesses of psychometric evaluations such as first-generation scales (BPRS, SANS, PANSS, NSA) and secondgeneration instruments (BNSS CAINS and MAP-SR). Especially with respect to assessment of primary negative symptoms, further research is needed. Since effective treatment of negative symptoms is an unmet need in the therapy of schizophrenia, Möller and Czobor [3] with focus on results of meta-analyses, review psychopharmacological treatment options including first- and second-generation antipsychotics. Modern antidepressants and add-on treatment with glutamatergic compounds have demonstrated some efficacy, but results are inconsistent and further research is needed to identify effective treatment of this symptom domain.

Andrea Schmitt

Andrea.Schmitt@med.uni-muenchen.de

1 Department of Psychiatry and Psychotherapy, LudwigMaximilians-University Munich, Nußbaumstr. 7, 80336 Munich, Germany
Because only few data are available on efficacy and safety of dopamine D2 receptor blocking antipsychotics, in a prospective, open-label, randomized study, Takekita et al. [4] investigated a 12-week lasting treatment with the full D2 receptor antagonist perospirone or the partial D2 receptor agonist aripiprazole in antipsychotic-naïve and antipsychotic-treated schizophrenia patients. Antipsychotic-naïve patients with perospirone treatment had greater symptom improvement compared to the group with previous antipsychotic treatment, whereas in patients receiving aripiprazole no difference in efficacy was observed in both patient groups. Therefore, in patients receiving a full D2 receptor agonist, treatment history should be considered. New nonpharmacological treatment strategies of schizophrenia such as transcutaneous noninvasive vagus nerve stimulation (tVNS) have been developed. In a bicentric randomized, sham-controlled, double-blind clinical trial, Hasan et al. [5] investigated effects of 26-week tVNS of the left auricle in schizophrenia patients compared to a group receiving daily sham stimulation for 12 weeks followed by 14 weeks of active stimulation. While the intervention was well tolerated with no relevant adverse effects, no improvement in schizophrenia symptoms could be observed during the observation period of 12 weeks. Further studies in larger samples are needed to draw conclusions on efficacy of this new brain stimulation method in schizophrenia.

The development of new therapeutic strategies in schizophrenia depends on improvement in the understanding of the neurobiological basis of the disorder. In a proteomic study of post-mortem tissue from the corpus callosum, which is a white matter region connecting the two brain hemispheres, Saia-Cereda et al. [6] collected cytoplasmatic, soluble proteins and performed nano-liquid mass spectroscopy in nine schizophrenia patients compared to seven healthy controls. They found 1636 proteins with 65 being 
differentially expressed, among them proteins associated with cell growth and maintenance, such as neurofilaments and tubulin, cell communication and oligodendrocyte function such as myelin basic protein and myelin-oligodendrocyte glycoprotein. Since 30 of these proteins had been found to be differentially expressed in previous postmortem studies of other brain regions, they may be markers of underlying pathophysiology and disconnectivity in schizophrenia.

A consequence of disturbed connectivity may be impaired cognition and psychosocial functioning. Deficits in processing and interpretation of social information from facial expression are features of schizophrenia symptomatology. Radke et al. [7] assessed social motivation to facial expressions and emotion recognition abilities in schizophrenia patients. Compared to healthy controls, patients showed less pronounced approach-avoidance ratings to happy and angry facial expressions along with prolonged reactions during automatic approach-avoidance. However, these deficits were not associated with alterations in social motivation. Since only little is known about the interaction of deficits in empathy and cognition in schizophrenia, $\mathrm{Hu}$ et al. [8] investigated effects of empathetic pain observation on cognition in schizophrenia patients. The group of healthy controls showed increased Stroop facilitation and decreased interference during the empathetic pain condition compared with the non-empathetic condition. Schizophrenia patients demonstrated a similar empathy effect on Stroop facilitation, but a larger empathy effect on Stroop interference, along with deficits in cognition. In the patient group, general rather than specific cognitive deficits are suggested and at least some components of empathetic pain processing are shown to be preserved.

\section{References}

1. Galderisi S, Merlotti E, Mucci A (2015) Neurobiological background of negative symptoms. Eur Arch Psychiatry Clin Neurosci. doi:10.1007/s00406-015-0590-4

2. Garcia-Portilla MP, Garcia-Alvarez L, Saiz PA, Al-Halab S, Bobes-Bascaran MT, Bascaran MT, Muñiz J, Bobes J (2015) Psychometric evaluation of the negative syndrome of schizophrenia. Eur Arch Psychiatry Clin Neurosci. doi:10.1007/ s00406-015-0595-Z

3. Möller H-J, Czobor P (2015) Pharmacological treatment of negative symptoms in schizophrenia. Eur Arch Psychiatry Clin Neurosci. doi:10.1007/s00406-015-0596-y

4. Takekita Y, Fabbri C, Kato M, Nonen S, Sakai S, Sunada N, Koshikawa Y, Wakeno M, Okugawa G, Kinoshita T, Serretti A (2015) Antagonist and partial agonist at the dopamine D2 receptors in drug-naïve and non-drug-naïve schizophrenia: a randomized, controlled trial. Eur Arch Psychiatry Clin Neurosci. doi:10.1007/s00406-015-0605-1

5. Hasan A, Wolff-Menzler C, Pfeiffer S, Falkai P, Weidinger E, Jobst A, Hoell I, Malchow B, Yeganeh-Doost P, Strube W, Quast S, Müller N, Wobrock T (2015) Transcutaneous noninvasive vagus nerve stimulation (tVNS) in the treatment of schizophrenia: a bicentric randomized controlled pilot study. Eur Arch Psychiatry Clin Neurosci. doi:10.1007/s00406-015-0618-9

6. Saia-Cereda VM, Cassoli JS, Schmitt A, Falkai P, Nascimento JM, Martins-de-Souza D (2015) Proteomics of the corpus callosum unravel pivotal players in the dysfunction of cell signaling, structure, and myelination in schizophrenia brains. Eur Arch Psychiatry Clin Neurosci. doi:10.1007/s00406-015-0621-1

7. Radke S, Pfersmann V, Derntl B (2015) The impact of emotional faces on social motivation in schizophrenia. Eur Arch Psychiatry Clin Neurosci. doi:10.1007/s00406-015-0589-x

8. Hu K, Lijffijt M, Beauchaine TP, Fan Z, Shi H, He S (2014) Influence of empathetic pain processing on cognition in schizophrenia. Eur Arch Psychiatry Clin Neurosci. doi:10.1007/ s00406-014-0565-x 